\title{
Caracterización de pacientes con hepatitis C crónica tratados en un hospital de alta complejidad de Medellín
}

\section{Characterization of patients with chronic hepatitis C treated in a high complexity hospital in Medellín}

\author{
Mónica Ledezma-Morales, ${ }^{1 *}$ Juan Carlos Restrepo G., ${ }^{2}$ Pedro Amariles, ${ }^{1}$ María Camila Trillos A., ${ }^{3}$ Rubén Darío Vargas R. ${ }^{3}$
}

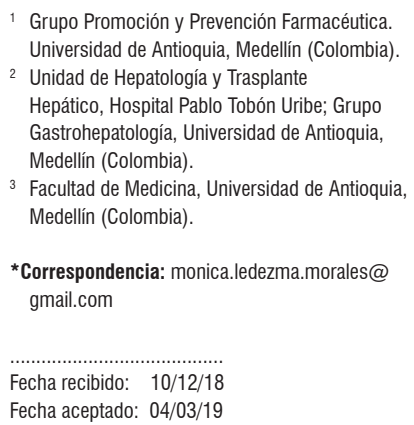

\begin{abstract}
Resumen
Introducción: la hepatitis $\mathrm{C}(\mathrm{HepC})$ representa un problema de salud pública a nivel mundial. Se estima que en Colombia la prevalencia de virus de la hepatitis C (VHC) está entre el 0,5-1\%, y asciende al 2,1\% en pacientes mayores de 50 años. La Unidad de Hepatología del Hospital Pablo Tobón Uribe (HPTU) ha sido un referente en el manejo de la HepC en Medellín y Colombia durante años. Objetivo: describir las características sociodemográficas/clínicas y los resultados en salud de los pacientes con HepC crónica atendidos en el HPTU entre 2013 y 2018. Materiales y métodos: estudio observacional, descriptivo, retrospectivo de pacientes con HepC crónica atendidos entre el 1 de enero de 2013 y el 31 de marzo de 2018. Resultados: se analizaron 108 pacientes. La edad promedio fue de 55,8 años (desviación estándar [DE] 13,7), 51,9\% eran hombres, y $78,7 \%$ pertenecían al régimen contributivo. El mecanismo de transmisión más frecuente fue la hemotransfusión; el genotipo 1 predominó en el grupo de pacientes analizados. La efectividad de los esquemas con interferón fue del 46,9\% y de los antivirales de acción directa (AAD) del 94,6 \%. La presencia de reacciones adversas a medicamentos (RAM) fue del 68,2 \% en pacientes con esquemas con interferón/ ribavirina y del 25,9\% en pacientes con AAD. Conclusiones: se realiza la caracterización de los pacientes atendidos en el HPTU, en quienes los AAD han mostrado mayor efectividad y seguridad en comparación con esquemas con interferón/ribavirina.
\end{abstract}

\section{Palabras clave}

Hepatitis C, Colombia, antivirales, interferones, antivirales de acción directa.

\begin{abstract}
Introduction: Throughout the world hepatitis $\mathrm{C}(\mathrm{HepC})$ is a public health problem. Estimates for its prevalence in Colombia range from $0.5 \%$ to $1 \%$ but $2.1 \%$ for patients over 50 years of age. The Hepatology Unit at the Hospital Pablo Tobón Uribe (HPTU) has been a benchmark for management of HepC in Medellín and Colombia for years. Objective: To describe sociodemographic and clinical characteristics together with health outcomes of patients with chronic HepC who were treated at the HPTU between 2013 and 2018. Materials and methods: This is an observational, descriptive and retrospective study of patients with chronic HepC, treated between January 1, 2013 and March 31, 2018. Results: One hundred and eight patients were analyzed. The average age was 55.8 years (SD 13.7), $51.9 \%$ were men, and $78.7 \%$ belonged to the contributory health care scheme. Most frequently, the disease was transmitted by blood, and genotype 1 predominated in the group of patients analyzed. The effectiveness of interferon schemes was $46.9 \%$ while that of Direct-Acting Antivirals (DAA) was $94.6 \%$. Adverse drug reactions were found in $68.2 \%$ of patients treated with interferon/ ribavirin schemes but in only $25.9 \%$ of the patients treated with DAA. Conclusions: In this group of patients treated at HPTU, DAA were safer and more effective than interferon/ribavirin schemes.
\end{abstract}

\section{Keywords}

Hepatitis C, Colombia, antivirals, interferons, direct action antivirals. 


\section{INTRODUCCIÓN}

La Hepatitis C (HepC) es un problema de salud pública mundial por su prevalencia (2-3\%), progresión a la cronicidad (70-90\% de los pacientes desarrolla enfermedad hepática crónica: cirrosis y carcinoma hepatocelular $[\mathrm{CHC}])(1$, 2), y afectación de poblaciones vulnerables, como usuarios de drogas inyectables y personas con asistencia sanitaria inadecuada. En Colombia, se estima que la prevalencia de la HepC está entre 0,5-1\%, y asciende al 2,1 \% en mayores de 50 años (3).

El objetivo del tratamiento es reducir la mortalidad por cualquier causa y las consecuencias adversas para la salud relacionadas con el hígado, incluyendo la enfermedad hepática terminal y el CHC; mediante el alcance de la respuesta virológica sostenida (RVS) (4), definida como una carga viral indetectable medida a las 12 o 24 semanas después del final del tratamiento en terapias libres de interferón o terapias basadas en interferón, respectivamente (5).

El tratamiento de la $\mathrm{HepC}$ ha evolucionado considerablemente, y ha reemplazado a las terapias con interferón pegilado (peg-IFN) y ribavirina (RBV), que presentaban poca tolerabilidad y RVS entre 6-56 $\%(2,6)$, por los antivirales de acción directa (AAD), que ofrecen tasas superiores de RVS (mayores al $90 \%$ ) (7), disminución del tiempo de tratamiento y reducción de eventos adversos $(5,8)$. En los Estados Unidos se aprobaron los $\mathrm{AAD}$ de segunda generación en el 2013; después se utilizaron en Colombia como vitales no disponibles, para luego comenzar la comercialización de simeprevir (SMV), daclatasvir (DCV) y asunaprevir en el 2015, seguidos del paritaprevir/ombitasvir/ ritonavir/dasabuvir (PrOD) en el 2016 y, de sofosbuvir (SOF) y ledipasvir (LDV) en 2017 (9).

El Hospital Pablo Tobón Uribe (HPTU), como centro de referencia en hepatología, ha sido responsable del manejo de pacientes con HepC crónica de diversos lugares de Colombia; sin embargo, allí no se cuenta con información sistematizada sobre sus características. Por lo tanto, el objetivo del presente trabajo fue describir las características sociodemográficas/ clínicas y los resultados en salud de los pacientes con HepC atendidos en el HPTU entre 2013 y 2018.

\section{MATERIAL Y MÉTODOS}

\section{Tipo de estudio}

Estudio observacional, descriptivo, retrospectivo.

\section{Población de estudio}

Pacientes con HepC crónica (diagnóstico confirmado por presencia de ARN del virus de la hepatitis $\mathrm{C}[\mathrm{VHC}]$ ), aten- didos en el HPTU entre el 1 de enero de 2013 y el 31 de marzo de 2018. Se excluyeron los pacientes sin tratamiento farmacológico entre las fechas analizadas, tratados antes del 2013 o con información incompleta de tratamiento.

\section{Variables}

- Sociodemográficas: sexo, edad, escolaridad, aseguradora, régimen de afiliación, departamento de residencia.

- Clínicas: mecanismo de transmisión del VHC, genotipo/subtipo delVHC, estado de fibrosis/cirrosis, coinfección con el virus de inmunodeficiencia humana (VIH) y/o virus de la hepatitis B (VHB), esquema tratamiento previo, tratamiento con $\mathrm{AAD}$, variantes asociadas a la resistencia (VAR), reacción adversa a medicamentos (RAM), número de medicamentos no anti-VHC utilizados por el paciente, y hospitalización en el HPTU relacionada con la HepC, y la RVS.

\section{Proceso de recolección de la información}

Se solicitó el consolidado de pacientes con códigos CIE-10 B182 y B171, el cual se completó con pacientes reportados por el hepatólogo investigador. Se extrajeron las variables sociodemográficas/clínicas de la historia clínica electrónica (HCE), y se registraron en un formulario en Microsoft Access $^{\circledast} 2010$.

\section{Análisis estadístico}

Para las variables cualitativas se utilizaron frecuencias absolutas y frecuencias relativas, y para las variables cuantitativas, media y desviación estándar. El análisis estadístico de los datos se realizó con SPSS $23^{\odot}$.

\section{Consideraciones éticas}

Se recibió la aprobación por parte del Comité de Investigaciones y Ética en la Investigación del HPTU (Protocolo 2018.033).

\section{RESULTADOS}

En el análisis se incluyeron 108 pacientes (Figura 1); un $51,9 \%$ eran hombres, y la edad promedio fue de 55,8 años (desviación estándar [DE] 13,7) (Tabla 1). El mecanismo de transmisión más frecuente fue la transfusión de hemoderivados (25\%), y el genotipo 1 tuvo mayor prevalencia (77,8 \%). El 39,8 \% de los pacientes presentaban fibrosis avanzada/cirrosis (F3-F4) y el 77,5\% de los pacientes en F4 tenían cirrosis compensada. El 4,6 \% de los pacientes tenía reporte de $\mathrm{CHC}$; el 90,7 \% no presentaba coinfeccio- 


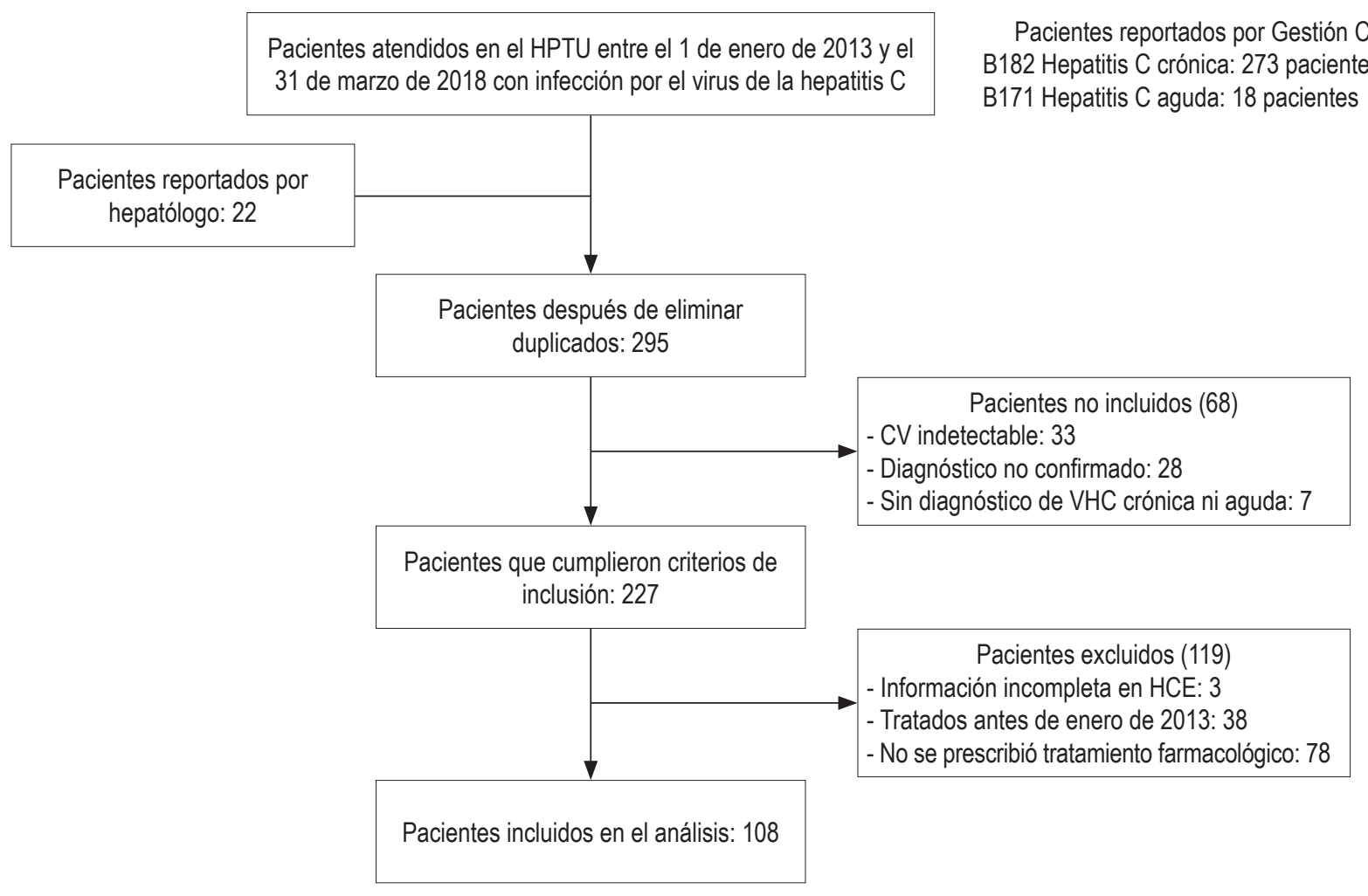

Figura 1. Diagrama general de la investigación. CV: carga viral; HCE: historia clínica electrónica; HPTU: Hospital Pablo Tobón Uribe.

Tabla 1. Características sociodemográficas de los pacientes con hepatitis C crónica

\begin{tabular}{|c|c|c|c|c|c|}
\hline Características & Frecuencia & $\%(n=108)$ & Características & Frecuencia & $\%(n=108)$ \\
\hline Sexo & & & Régimen de seguridad social & & \\
\hline Hombre & 56 & 51,9 & Subsidiado & 11 & 10,2 \\
\hline Mujer & 52 & 48,1 & Contributivo & 85 & 78,7 \\
\hline Edad & & & Excepción & 6 & 5,6 \\
\hline$\leq 30$ & 5 & 4,6 & Particular & 2 & 1,9 \\
\hline $31-40$ & 12 & 11,1 & No reporta & 4 & 3,7 \\
\hline $41-50$ & 15 & 13,9 & \multicolumn{3}{|c|}{ Empresa administradora de planes de beneficios } \\
\hline $51-60$ & 32 & 29,6 & SURA EPS & 24 & 22,2 \\
\hline $61-70$ & 30 & 27,8 & Nueva EPS & 19 & 17,6 \\
\hline $71-80$ & 12 & 11,1 & Coomeva EPS & 14 & 13,0 \\
\hline$>81$ & 2 & 1,9 & Otras & 50 & 46,3 \\
\hline Escolaridad & & & No reporta & 1 & 0,9 \\
\hline Básica primaria & 12 & 11,1 & \multicolumn{3}{|l|}{ Departamento de residencia } \\
\hline Básica secundaria & 23 & 21,3 & Antioquia & 86 & 79,6 \\
\hline Técnica profesional & 5 & 4,6 & Atlántico & 5 & 4,6 \\
\hline Tecnológica & 2 & 1,9 & Risaralda & 5 & 4,6 \\
\hline Profesional & 24 & 22,2 & Quindío & 3 & 2,8 \\
\hline Posgrado & 3 & 2,8 & Cundinamarca & 2 & 1,9 \\
\hline No reporta & 39 & 36,1 & Otros & 7 & 6,5 \\
\hline
\end{tabular}


nes y el 31,5\% tuvo hospitalización en el HPTU por causas relacionadas con la HepC. Las demás características clínicas pueden observarse en la Tabla 2.

\section{Tratamiento de la infección por VHC}

El $37 \%$ de los pacientes fue tratado únicamente con terapias con peg-INF, el $24,1 \%$ con esquemas con peg-INF con posterior prescripción de terapia de rescate con $\mathrm{AAD}$, y al $38,9 \%$ solo se le prescribió tratamiento con AAD (Tabla 3).

Tabla 2. Características clínicas de los pacientes con hepatitis C crónica

\begin{tabular}{|c|c|c|}
\hline & Frecuencia & $\begin{array}{c}\% \\
\text { (n }=108\end{array}$ \\
\hline \multicolumn{3}{|l|}{ Posible mecanismo de transfusión } \\
\hline Hemotransfusión & 27 & 25,0 \\
\hline Transmisión sexual & 8 & 7,4 \\
\hline $\begin{array}{l}\text { Uso de equipos de inyección } \\
\text { contaminados (persona que se inyecta } \\
\text { drogas psicoactivas) }\end{array}$ & 7 & 6,5 \\
\hline $\begin{array}{l}\text { Evento adverso relacionado con } \\
\text { procedimientos de salud }\end{array}$ & 4 & 3,7 \\
\hline Exposición ocupacional & 1 & 0,9 \\
\hline $\begin{array}{l}\text { Hemotransfusión y otras formas de } \\
\text { transmisión sanguínea (tatuajes, piercings, } \\
\text { escarificaciones) }\end{array}$ & 1 & 0,9 \\
\hline $\begin{array}{l}\text { Otras formas de transmisión sanguínea } \\
\text { (tatuajes, piercings, escarificaciones) }\end{array}$ & 1 & 0,9 \\
\hline Transmisión materno infantil & 1 & 0,9 \\
\hline Desconocido & 58 & 53,7 \\
\hline \multicolumn{3}{|l|}{ Genotipo } \\
\hline 1 & 7 & 6,5 \\
\hline $1 a$ & 33 & 30,6 \\
\hline $1 a-1 b$ & 1 & 0,9 \\
\hline $1 b$ & 43 & 39,8 \\
\hline 2 & 7 & 6,5 \\
\hline $2 a$ & 1 & 0,9 \\
\hline $2 b$ & 2 & 1,9 \\
\hline 3 & 2 & 1,9 \\
\hline 4 & 3 & 2,8 \\
\hline No genotipificado & 9 & 8,3 \\
\hline \multicolumn{3}{|l|}{ Coinfección } \\
\hline $\mathrm{VIH}$ & 7 & 6,5 \\
\hline VHB & 3 & 2,8 \\
\hline Ninguna & 98 & 90,7 \\
\hline \multicolumn{3}{|l|}{ Estado de trasplante hepático } \\
\hline Sin trasplante & 91 & 84,3 \\
\hline
\end{tabular}

Del total de pacientes que recibió terapias con peg-INF $(61,1 \%)$, el $59,1 \%$ recibió boceprevir o telaprevir. El $46,9 \%$ de los pacientes que recibió peg-INF alcanzó RVS (Figura 2). En 5 pacientes no se reportó RVS, 3 pacientes quedaron a la espera de terapias libres de interferón y un paciente falleció debido a un choque séptico de origen urinario y una encefalopatía hepática grave. A 26 pacientes que no alcanzaron la curación se les prescribieron $\mathrm{AAD}$.

El 68,2\% de los pacientes con peg-IFN tenía reporte de RAM en la HCE, los esquemas con boceprevir en mayor

\begin{tabular}{|c|c|c|}
\hline & Frecuencia & $\begin{array}{c}\% \\
(n=108)\end{array}$ \\
\hline \multicolumn{3}{|l|}{ Estado de trasplante hepático } \\
\hline Trasplantado & 16 & 14,8 \\
\hline Trasplante previo y en lista de trasplante & 1 & 0,9 \\
\hline \multicolumn{3}{|l|}{ Estado de fibrosis } \\
\hline No especificada & 20 & 18,5 \\
\hline F0 & 8 & 7,4 \\
\hline F1 & 15 & 13,9 \\
\hline F1-2 & 3 & 2,8 \\
\hline F2 & 12 & 11,1 \\
\hline F2-3 & 3 & 2,8 \\
\hline F3 & 4 & 3,7 \\
\hline F3-4 & 3 & 2,8 \\
\hline F4 (Cirrosis) & 40 & 37,0 \\
\hline \multicolumn{3}{|l|}{ Cirrosis (Child-Pugh-Turcotte) } \\
\hline Compensada (A) & 31 & 77,5 \\
\hline No compensada (B) & 6 & 15,0 \\
\hline No compensada (C) & 2 & 5,0 \\
\hline No clasificada & 1 & 2,5 \\
\hline \multicolumn{3}{|l|}{ Carcinoma hepatocelular } \\
\hline Sí & 5 & 4,6 \\
\hline No & 101 & 93,5 \\
\hline Sospecha & 1 & 0,9 \\
\hline No reporta & 1 & 0,9 \\
\hline \multicolumn{3}{|l|}{ Manifestaciones extrahepáticas } \\
\hline Dermatológicas & 9 & 8,3 \\
\hline Hematológicas & 5 & 4,6 \\
\hline Desórdenes autoinmunes & 3 & 2,8 \\
\hline Renales & 2 & 1,9 \\
\hline Ninguna & 89 & 82,4 \\
\hline \multicolumn{3}{|l|}{ Hospitalización en HPTU asociada a VHC } \\
\hline Sí & 34 & 31,5 \\
\hline No & 74 & 68,5 \\
\hline
\end{tabular}


Tabla 3. Esquemas de tratamiento prescritos para el manejo de la hepatitis $\mathrm{C}$

\begin{tabular}{|c|c|c|c|c|c|c|}
\hline \multirow[t]{2}{*}{ Antivirales acción directa } & \multicolumn{5}{|c|}{ Esquemas con interferón } & \multirow[b]{2}{*}{ Total genera } \\
\hline & $\begin{array}{c}\text { BOC/peg-INF/ } \\
\text { RBV }\end{array}$ & peg-INF/RBV & $\begin{array}{c}\text { TPV/peg-INFI } \\
\text { RBV }\end{array}$ & peg-INF & Ninguno & \\
\hline Ninguno & 24 & 10 & 5 & 1 & & 40 \\
\hline SOF/LDV & & & & & 12 & 12 \\
\hline SOF/DCV/RBV & 3 & 4 & 1 & & 4 & 12 \\
\hline SOF/DCV & & 1 & 1 & & 7 & 9 \\
\hline PTV/OBV/R/Dasabuvir /RBV & & & & 1 & 8 & 9 \\
\hline PTV/OBV/R/Dasabuvir & & 3 & & 1 & 5 & 9 \\
\hline DCV/Asunaprevir & 1 & 2 & 1 & & 4 & 8 \\
\hline SOF/LDV/RBV & 1 & 3 & 1 & & 2 & 7 \\
\hline SOF/SMV/RBV & & 1 & & & & 1 \\
\hline SOF/RBV & 1 & & & & & 1 \\
\hline Total general & 30 & 24 & 9 & 3 & 42 & 108 \\
\hline
\end{tabular}

BOC: boceprevir; DCV: daclatasvir; LDV: ledipasvir; OBV: ombitasvir; peg-IFN: interferón pegilado; PTV: paritaprevir; R: ritonavir; RBV: ribavirina; SMV: simeprevir; SOF: sofosbuvir; TPV: telaprevir.

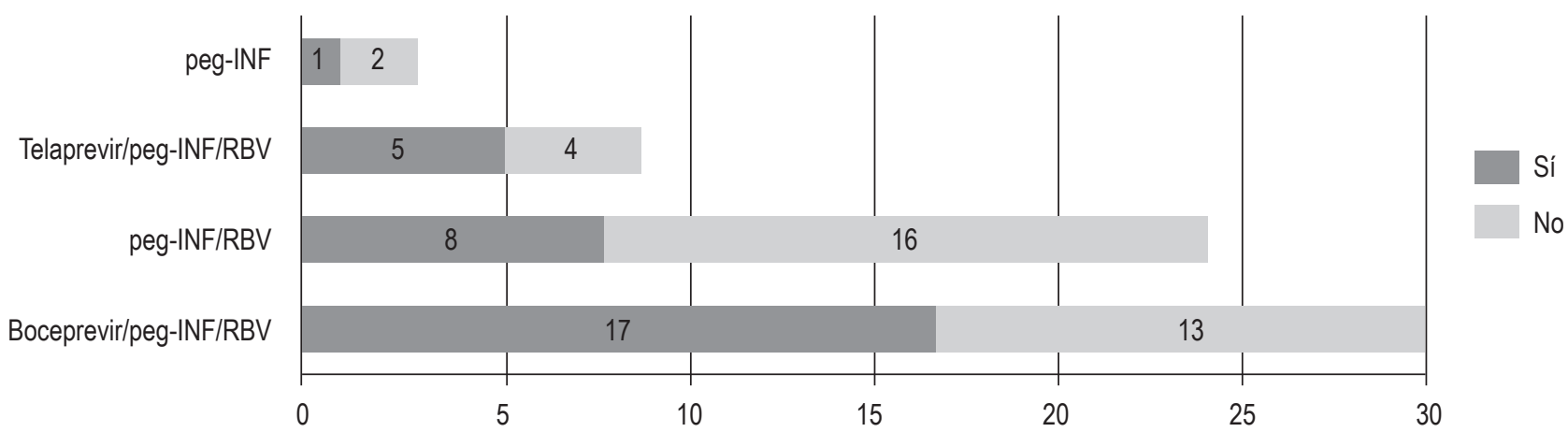

Figura 2. Alcance de respuesta virológica sostenida con interferón ( $\mathrm{n}=66)$. peg-INF: Interferón pegilado; RBV: ribavirina.

frecuencia. Se registraron en total 216 RAM, siendo más frecuentes la astenia y la neutropenia ( $8,8 \%$ cada una), la anemia $(7,9 \%)$, la leucopenia y la adinamia $(6,9 \%$ cada una) (Tabla 4).

\section{Uso de antivirales de acción directa}

Los AAD más prescritos fueron SOF/LDV y SOF/DCV/ RBV (Tabla 3). El 79,4 \% de los pacientes con prescripción de AAD tiene reporte de inicio de tratamiento, de los cuales el 88,9\% lo finalizó (Figura 3). De los pacientes que finalizaron tratamiento, el 77,1 \% (37/48) tenía reporte de carga viral a las 12 semanas después del fin de tratamiento, de los cuales el 94,6 \% alcanzó RVS (Figura 4). El 5,4 \% restante no logró RVS por existencia de VAR, principalmente a inhibidores de la NS5A. La primera paciente estuvo expuesta a DCV/asunaprevir por 24 semanas sin logro de RVS; para este caso no se inició otro esquema dados los costos y riesgos de efectos colaterales. El segundo paciente recibió SOF/SMV/RBV por 12 semanas, pero no alcanzó RVS, y el médico especialista reportó la no disponibilidad de tratamiento. Cabe resaltar que una tercera paciente presentó VAR con alcance de RVS (Tabla 5).

$\mathrm{El}$ análisis de la seguridad de los $\mathrm{AAD}$ se realizó con los pacientes que tenían un reporte de inicio de tratamiento; el $25,9 \%(14 / 54)$ tenían registro de RAM asociadas a AAD. Se identificaron $37 \mathrm{RAM}$ atribuidas a 7 esquemas de $\mathrm{AAD}$, siendo SOF/DCV/RBV el de mayor frecuencia, seguido 
Tabla 4. Reacciones adversas a esquemas con interferón, ribavirina e inhibidores de proteasa (boceprevir o telaprevir)

\begin{tabular}{|c|c|c|c|c|c|}
\hline \multirow{2}{*}{ RAM } & \multicolumn{3}{|c|}{ Medicamento } & \multirow{2}{*}{$\mathrm{n}$} & \multirow{2}{*}{$\%$} \\
\hline & $\mathrm{BOC} /$ peg-INF/RBV & peg-INF/RBV & TPV/ peg-INF/RBV & & \\
\hline Sistémicas & 37 & 42 & 7 & 86 & 39,8 \\
\hline Astenia & 8 & 9 & 2 & 19 & 8,8 \\
\hline Adinamia & 7 & 7 & 1 & 15 & 6,9 \\
\hline Fiebre & 5 & 5 & 1 & 11 & 5,1 \\
\hline Dolor muscular & 2 & 6 & & 8 & 3,7 \\
\hline Cefalea & 4 & 2 & & 6 & 2,8 \\
\hline Malestar general & 2 & 4 & & 6 & 2,8 \\
\hline Hiporexia & 3 & 2 & & 5 & 2,3 \\
\hline Escalofríos & 2 & 1 & 1 & 4 & 1,9 \\
\hline Tos & 1 & 2 & & 3 & 1,4 \\
\hline Síntomas gripales & 1 & 1 & & 2 & 0,9 \\
\hline Odinofagia & & 1 & & 1 & 0,5 \\
\hline Polimiositis con elevación de CK & & 1 & & 1 & 0,5 \\
\hline Rinorrea & & 1 & & 1 & 0,5 \\
\hline Disnea & & & 1 & 1 & 0,5 \\
\hline Síntomas respiratorios & 1 & & & 1 & 0,5 \\
\hline Sinusitis & & & 1 & 1 & 0,5 \\
\hline Debilidad & 1 & & & 1 & 0,5 \\
\hline Hematológicas & 36 & 9 & 12 & 57 & 26,4 \\
\hline Neutropenia & 12 & 4 & 3 & 19 & 8,8 \\
\hline Anemia & 13 & 1 & 3 & 17 & 7,9 \\
\hline Leucopenia & 8 & 3 & 4 & 15 & 6,9 \\
\hline Trombocitopenia & 2 & & 1 & 3 & 1,4 \\
\hline Pancitopenia & 1 & & 1 & 2 & 0,9 \\
\hline Alteraciones hematológicas & & 1 & & 1 & 0,5 \\
\hline Gastrointestinales & 10 & 9 & 2 & 21 & 9,7 \\
\hline Náuseas & 3 & 2 & & 5 & 2,3 \\
\hline Epigastralgia & 3 & & & 3 & 1,4 \\
\hline Reflujo gastroesofágico & 1 & 2 & & 3 & 1,4 \\
\hline Vómito & 2 & 1 & & 3 & 1,4 \\
\hline Diarrea & 1 & & 1 & 2 & 0,9 \\
\hline Pérdida del apetito & & 2 & & 2 & 0,9 \\
\hline Dispepsia & & 1 & & 1 & 0,5 \\
\hline Eructos & & 1 & & 1 & 0,5 \\
\hline Sintomas gastrointestinales & & & 1 & 1 & 0,5 \\
\hline Neuropsiquiátricas & 11 & 8 & & 19 & 8,8 \\
\hline Depresión & 6 & 4 & & 10 & 4,6 \\
\hline Insomnio & 1 & 1 & & 2 & 0,9 \\
\hline Disgeusia & 1 & & & 1 & 0,5 \\
\hline Hipersomnia & & 1 & & 1 & 0,5 \\
\hline Hipomanía & & 1 & & 1 & 0,5 \\
\hline Ideación suicida & 1 & & & 1 & 0,5 \\
\hline Ansiedad & & 1 & & 1 & 0,5 \\
\hline Irritabilidad & 1 & & & 1 & 0,5 \\
\hline Vértigo & 1 & & & 1 & 0,5 \\
\hline
\end{tabular}


Tabla 4. Reacciones adversas a esquemas con interferón, ribavirina e inhibidores de proteasa (boceprevir o telaprevir) (continuación)

\begin{tabular}{|c|c|c|c|c|c|}
\hline \multirow{2}{*}{ RAM } & \multicolumn{3}{|c|}{ Medicamento } & \multirow{2}{*}{$\mathrm{n}$} & \multirow{2}{*}{$\%$} \\
\hline & $\mathrm{BOC} / \mathrm{peg}-\mathrm{INF} / \mathrm{RBV}$ & peg-INF/RBV & TPV/ peg-INF/RBV & & \\
\hline Dermatológicas & 9 & 2 & 6 & 17 & 7,9 \\
\hline Prurito & 4 & & 2 & 6 & 2,8 \\
\hline Rash & 2 & 1 & 3 & 6 & 2,8 \\
\hline Alopecia & 3 & & & 3 & 1,4 \\
\hline Lesiones en piel & & 1 & 1 & 2 & 0,9 \\
\hline Misceláneos & 4 & 4 & 4 & 12 & 5,6 \\
\hline Dolor anal & & & 3 & 3 & 1,4 \\
\hline Aftas orales & & & 1 & 1 & 0,5 \\
\hline Aumento de peso & 1 & & & 1 & 0,5 \\
\hline Disfonia & & 1 & & 1 & 0,5 \\
\hline Disminución de peso & & 1 & & 1 & 0,5 \\
\hline Dolor pleurítico & & 1 & & 1 & 0,5 \\
\hline Fosfenos & 1 & & & 1 & 0,5 \\
\hline Hemoptisis & & 1 & & 1 & 0,5 \\
\hline Hiperbilirrubinemia & 1 & & & 1 & 0,5 \\
\hline Sangrado hemorroidal & 1 & & & 1 & 0,5 \\
\hline Endocrinas & 1 & 2 & 1 & 4 & 1,9 \\
\hline Alteraciones tiroideas & & 2 & 1 & 3 & 1,4 \\
\hline Aumento de glucemia & 1 & & & 1 & 0,5 \\
\hline Total general & 108 & 76 & 32 & 216 & 100,0 \\
\hline
\end{tabular}

BOC: boceprevir; peg-INF: interferón pegilado; RAM: reacción adversa a medicamento; RBV: ribavirina; TPV: telaprevir.

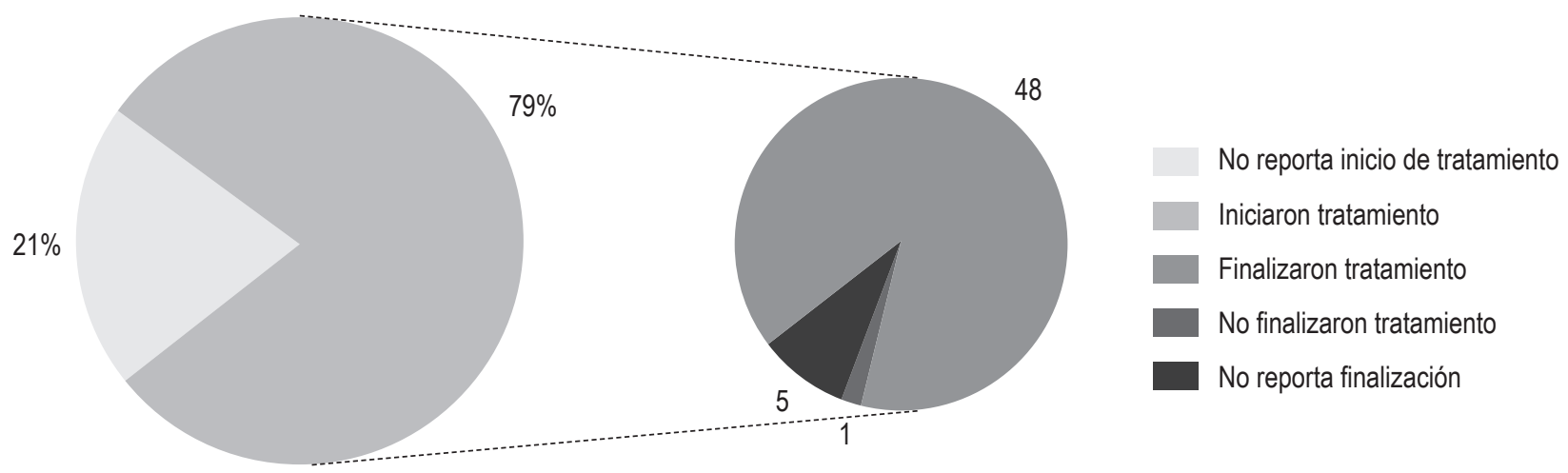

Figura 3. Estado del tratamiento con antivirales de acción directa $(n=68)$.

de SOF/LDV/RBV. Las RAM más frecuentes fueron anemia $(16,2 \%)$, astenia $(10,8 \%)$, cefalea y síntomas gripales ( $8,1 \%$ cada una) (Tabla 6). Ninguna de las RAM reportadas ocasionó suspensión del tratamiento.

\section{Polifarmacia en pacientes con HepC}

El 46,3 \% de los pacientes utilizaba cuatro o más medicamentos diferentes a los esquemas anti-VHC (Tabla 7). 


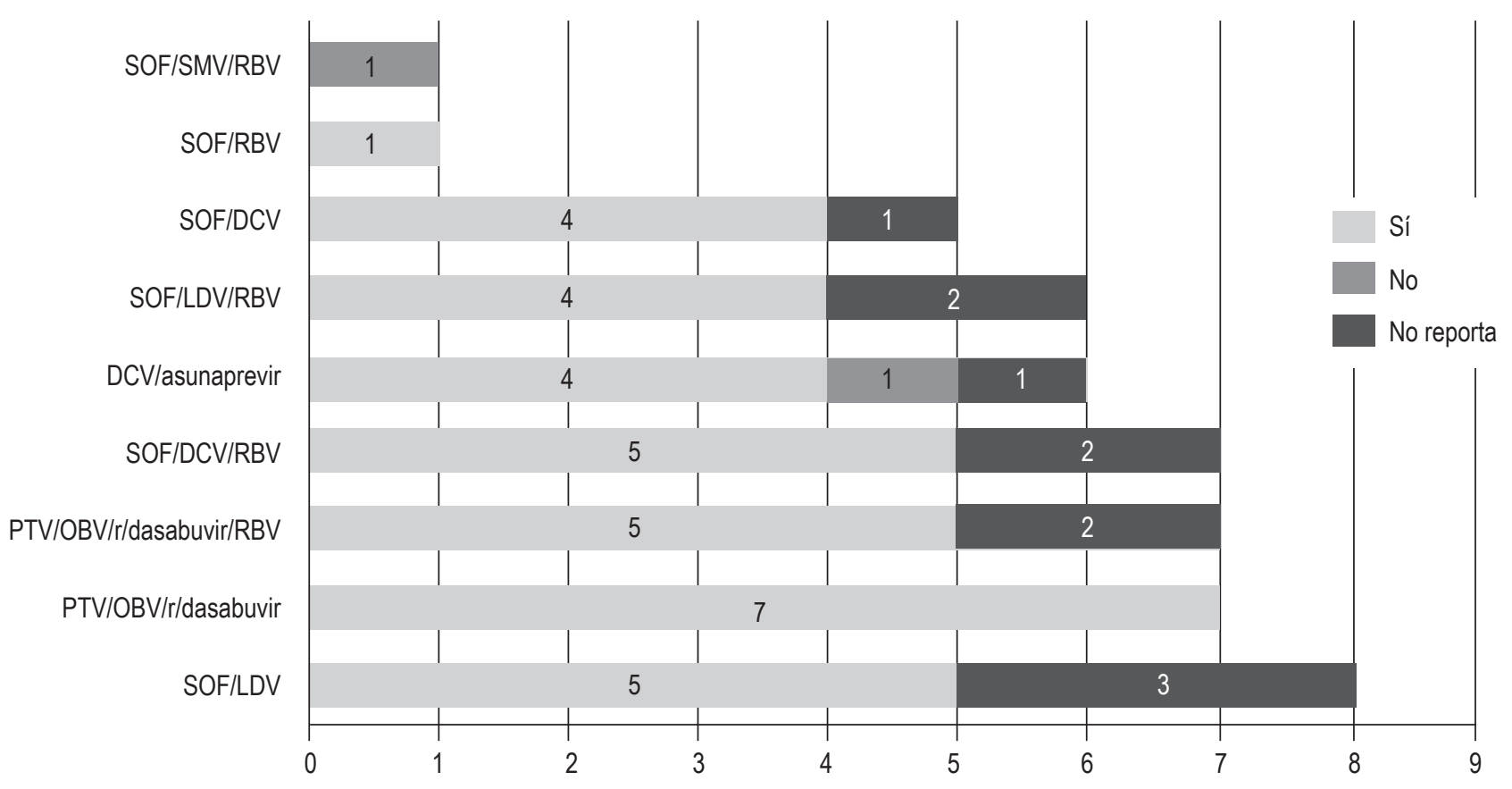

Figura 4. Alcance de respuesta viral sostenida con esquemas Antivirales de Acción Directa (n=48). DCV: daclatasvir; LDV: ledipasvir; OBV: ombitasvir; PTV: paritaprevir; r: ritonavir; RBV: ribavirina; SMV: simeprevir; SOF: sofosbuvir.

Tabla 5. Pacientes con variantes asociadas a la resistencia

\begin{tabular}{|c|c|c|c|c|c|c|c|}
\hline & VAR & Sexo & Edad & GT & Esquema & Semanas & RVS \\
\hline 1 & L31V y Y93H: resistencia a DCV, EBV, LDV, OBV, VEL & Mujer & 78 & $1 \mathrm{~b}$ & DCV/asunaprevir & 24 & No \\
\hline 2 & Y93N: resistencia a DCV, ELB, LDV, OBV & Hombre & 78 & $1 a$ & SOF/SMV/RBV & 12 & No \\
\hline 3 & $\begin{array}{l}\text { L31V: resistencia a: DCV, EBV, LDV, OBV, susceptibilidad reducida a VEL } \\
\text { Q80K: resistencia a SMV. }\end{array}$ & Mujer & 35 & $1 a$ & SOF/LDV/RBV & 24 & Sí \\
\hline
\end{tabular}

DCV: daclatasvir; EBV: elbasvir; GT: genotipo; LDV: ledipasvir; OBV: ombitasvir; RBV: ribavirina; RVS: respuesta virológica sostenida; SMV: simeprevir; SOF: sofosbuvir; VAR: variante asociada a la resistencia; VEL: velpatasvir.

El 17,6 \% no contaba con registro de sus medicamentos ambulatorios.

\section{DISCUSIÓN}

Este estudio es un primer acercamiento al conocimiento de la población con HepC tratada en el HPTU y a los resultados de efectividad/seguridad de los $\mathrm{AAD}$ en esta población. La distribución de HepC por sexo y edad fue similar a la reportada por Santos y colaboradores, quienes a partir de 1538 muestras recolectadas en laboratorios de referencia en Colombia, encontraron una edad promedio de 53 años (DE 14), con aproximadamente el $70 \%$ de los pacientes entre los 40-70 años (10). El genotipo 1 y subtipo $1 \mathrm{~b}$ se encontraron en el 77,8 \% y el 39,8 \% de los pacientes anali- zados, respectivamente, lo que corresponde con los genotipos y subtipos predominantes en Colombia según Santos y colaboradores (10).

El 39,8\% de los pacientes presentaba fibrosis avanzada/ cirrosis (F3-F4), con predominio de cirrosis compensada en el 77,5\% de los casos en estadio F4. Un estudio transversal analítico realizado en Cartagena durante tres meses encontró que de 41 pacientes, el $50 \%$ de los sujetos presentaba cirrosis/fibrosis avanzada, y el $68 \%$ tenía cirrosis compensada (11). Estas diferencias pueden deberse al número de pacientes analizados y al corto tiempo de recolección de información de dicho estudio. La proporción de pacientes que presentaron cirrosis fue mayor en comparación con la descrita por Hajarizadeh y colaboradores (4-24\%) (1). Esto puede explicarse por el nivel de complejidad del 
Tabla 6. Reacciones adversas registradas por el esquema de tratamiento con antivirales de acción directa

\begin{tabular}{|c|c|c|c|c|c|c|c|c|c|}
\hline RAM Medicamento & $\begin{array}{c}\text { SOF/DCVI } \\
\text { RBV }\end{array}$ & $\begin{array}{l}\text { SOF/LDVI } \\
\text { RBV }\end{array}$ & $\begin{array}{c}\text { DCVI } \\
\text { asunaprevir }\end{array}$ & $\begin{array}{l}\text { PTV/OBV/rl } \\
\text { dasabuvir/ } \\
\text { RBV }\end{array}$ & $\begin{array}{l}\text { SOFI } \\
\text { DCV }\end{array}$ & $\begin{array}{l}\text { SOFI } \\
\text { LDV }\end{array}$ & $\begin{array}{c}\text { PTVI } \\
\text { OBV/rl } \\
\text { dasabuvir }\end{array}$ & $n$ & $\%$ \\
\hline Sistémicas & 7 & 3 & 4 & 1 & & & 1 & 16 & 43,2 \\
\hline Astenia & 2 & & 1 & & & & 1 & 4 & 10,8 \\
\hline Síntomas gripales & 1 & & 1 & 1 & & & & 3 & 8,1 \\
\hline Cefalea & 2 & 1 & & & & & & 3 & 8,1 \\
\hline Dolor en miembros inferiores & 1 & 1 & & & & & & 2 & 5,4 \\
\hline Síntomas inespecíficos constitucionales & & 1 & & & & & & 1 & 2,7 \\
\hline Artralgias & & & 1 & & & & & 1 & 2,7 \\
\hline Adinamia & & & 1 & & & & & 1 & 2,7 \\
\hline Mareo & 1 & & & & & & & 1 & 2,7 \\
\hline Hematológicas & 4 & 2 & & & & & & 6 & 16,2 \\
\hline Anemia & 4 & 2 & & & & & & 6 & 16,2 \\
\hline Neuropsiquiátricas & 2 & 1 & 1 & & 1 & 1 & & 6 & 16,2 \\
\hline Insomnio & 1 & & & & 1 & & & 2 & 5,4 \\
\hline Depresión & 1 & 1 & & & & & & 2 & 5,4 \\
\hline Irritabilidad & & & 1 & & & & & 1 & 2,7 \\
\hline Alteración de la memoria inmediata & & & & & & 1 & & 1 & 2,7 \\
\hline Gastrointestinales & 1 & 2 & & 2 & & & & 5 & 13,5 \\
\hline Diarrea & 1 & & & 1 & & & & 2 & 5,4 \\
\hline Náuseas & & 1 & & & & & & 1 & 2,7 \\
\hline Síntomas gastrointestinales & & & & 1 & & & & 1 & 2,7 \\
\hline Dispepsia & & 1 & & & & & & 1 & 2,7 \\
\hline Misceláneas & 1 & & & 1 & 1 & & & 3 & 8,1 \\
\hline Hipotensión & 1 & & & & & & & 1 & 2,7 \\
\hline Pérdida de peso & & & & & 1 & & & 1 & 2,7 \\
\hline Leve hiperbilirrubinemia indirecta & & & & 1 & & & & 1 & 2,7 \\
\hline Dermatológicas & & & 1 & & & & & 1 & 2,7 \\
\hline Prurito & & & 1 & & & & & 1 & 2,7 \\
\hline Total general & 15 & 8 & 6 & 4 & 2 & 1 & 1 & 37 & 100,0 \\
\hline
\end{tabular}

DCV: daclatasvir; LDV: ledipasvir; OBV: ombitasvir; PTV: paritaprevir; r: ritonavir; RAM: reacción adversa a medicamento; RBV: ribavirina; SMV: simeprevir; SOF: sofosbuvir.

HPTU, en el que generalmente se tratan a pacientes en estadios más avanzados de la enfermedad.

La transfusión de hemoderivados fue el principal factor de riesgo para contraer el $\mathrm{VHC}$, como lo reportan otros autores en Colombia y Latinoamérica $(12,13)$; estos son resultados esperables, puesto que en Colombia se inició el tamizaje de VHC en las donaciones de sangre en 1993, pero solamente hasta 1995 alcanzó una cobertura del $99 \%$ (14), y, considerando que la aparición de cirrosis comienza
Tabla 7. Polifarmacia en pacientes con hepatitis C crónica $(\mathrm{n}=108)$

\begin{tabular}{ccc}
\hline Número de medicamentos no anti-VHC & Frecuencia & $\%$ \\
\hline$<4$ & 39 & 36,1 \\
$4-7$ & 35 & 32,4 \\
$8-11$ & 13 & 12,0 \\
$>12$ & 2 & 1,9 \\
No reporta & 19 & 17,6 \\
\hline
\end{tabular}


20 años después de la exposición al VHC (1), durante los próximos años podría aumentar el número de diagnósticos de HepC como consecuencia de las transfusiones realizadas antes de 1993.

\section{Efectividad de la terapia antiviral}

El 46,9 \% de los pacientes con peg-IFN alcanzaron RVS, lo cual tiene relación con lo reportado en la literatura (6-56\%) (8); adicionalmente para el genotipo 1, predominante en el grupo analizado, la tasa de respuesta puede llegar hasta el $50 \%(15)$.

En el subgrupo de pacientes que finalizaron tratamiento con AAD se encontró una RVS del 94,6\%. Al 5,4 \% restante se les realizaron estudios de resistencia para analizar la causa del fallo terapéutico, y se encontró VAR de inhibidores de la NS5A. Estos hallazgos son congruentes con los de Buti y colaboradores, quienes afirmaron que el 1-7 \% de los pacientes tratados con AAD no alcanzan la RVS (16); esto podría ser atribuido a causas relacionadas con el paciente, el régimen del tratamiento y el virus (17).

Las VAR son cambios en la secuencia de nucleótidos encargada de la síntesis de proteínas que son blanco molecular de los $\mathrm{AAD}$; esta facultad de generar resistencia, propia de los virus, es mayor en el VHC que en otros virus como el VHB y VIH (17). Las VAR encontradas en este estudio fueron $\mathrm{L} 31 \mathrm{~V}$ y $\mathrm{Y} 93 \mathrm{H}$, dirigidas a los inhibidores de la NS5A. No se reportaron VAR del análogo de nucleótido NS5B sofosbuvir, y esto puede ser explicado por su alta barrera genética a la resistencia (18).

En este estudio, la tasa de RVS fue similar en pacientes con VAR a la NS5A y sin ellas, como ha sido reportado por otros autores (19). Algunos investigadores discrepan entre la relación de presentar VAR y la RVS, por lo que recomiendan determinar estas variantes de manera basal, especialmente en los casos que impliquen una respuesta nula previa a la terapia $(17,18)$. En Colombia, las guías actuales para el manejo de la HepC recomiendan hacer un análisis de resistencias a NS3 o NS5A únicamente en pacientes que no han alcanzado RVS (20).

Se han descrito que las VAR más frecuentes en el genotipo $1 \mathrm{~b}$ son $\mathrm{L} 31 \mathrm{~V} / \mathrm{M}$ y $\mathrm{Y} 93 \mathrm{H} / \mathrm{N}$, principalmente la $\mathrm{Y} 93 \mathrm{H}$ otorga una alta resistencia a los medicamentos que actúan sobre la NS5A. Resulta importante destacar que las VAR a NS5A se encuentran presentes incluso dos años tras finalizar el tratamiento y es indispensable considerarlas antes de administrar terapia de rescate (17).

\section{Seguridad de la terapia antiviral}

La disponibilidad de $\mathrm{AAD}$ ha supuesto una mejora en la tolerabilidad del tratamiento (8), como se observó en este estudio, donde el $25,9 \%$ de pacientes con $\mathrm{AAD}$ presentó alguna RAM en comparación con un $68,2 \%$ de aquellos con peg-INF. Aunque el análisis de gravedad de las RAM no fue objeto de este estudio, durante la recolección de datos pudo observarse que los pacientes con peg-INF/ RBV presentaron más RAM graves, principalmente hospitalizaciones por anemia con requerimiento de hemoderivados, e infecciones asociadas con leucopenia o neutropenia.

Se observó que el 39,8\% de los pacientes con peg-INF/ RBV presentaron RAM sistémicas, principalmente astenia, adinamia, fiebre, mialgia y cefalea, lo cual tiene relación con lo encontrado en la literatura, donde se describe que estos síntomas pueden presentarse entre el 11-50 \% de los casos (21-25), aparecen a las pocas horas después de la aplicación del medicamento, y tienen una remisión espontánea a las 24-48 horas (21) o varios días después (23). Las RAM hematológicas por peg-INF/RBV son las más comunes, $\mathrm{y}$ constituyen la principal causa de baja adherencia, disminución de dosis y suspensión del tratamiento $(21,22,26)$. En este estudio se presentaron en el $26,4 \%$ de los pacientes, con neutropenia y anemia, que podrían asociarse con la supresión de la medula ósea por peg-IFN y la hemólisis extravascular inducida por $\operatorname{RBV}(23,27)$.

En los pacientes tratados con $\mathrm{AAD}$, las RAM sistémicas fueron las de mayor ocurrencia $(43,2 \%)$, seguidas de las neuropsiquiátricas $(16,2 \%)$, hematológicas $(16,2 \%)$ y gastrointestinales $(13,5 \%)$. Barrajón y colaboradores presentaron resultados muy similares en un análisis retrospectivo de 355 pacientes tratados con $\mathrm{AAD}$, en donde el $43,7 \%$ presentó RAM, en su mayoría sistémicas $(37,1 \%)$, gastrointestinales $(18 \%)$ y neurológicas $(15,8 \%)(28)$. Se podría inferir que la aparición de RAM hematológicas y neuropsiquiátricas estuvo relacionada con el uso de RBV en combinación con SOF/DCV o SOF/LDV, siendo en ellos más frecuente el desarrollo de anemia y depresión, en comparación con quienes no usaron RBV. Calleja y colaboradores también mostraron una alta incidencia de anemia (91\%) en pacientes con SOF/LDV/RBV (7).

Estos resultados muestran que, aunque el uso de AAD aumenta la tolerabilidad al tratamiento antiviral, aún se presentan casos en los que es necesaria la adición de RBV o peg-INF, especialmente en pacientes expuestos previamente a interferón que presentan cirrosis $(4,20)$, lo cual aumenta el riesgo de aparición de RAM (28).

\section{Polifarmacia en pacientes con HepC}

La polifarmacia puede ser definida como el uso de cinco o más medicamentos diarios (29). En este estudio se encontró que el 46,3\% de los pacientes estaban polimedicados. Esta polifarmacia puede explicarse por la edad ( $>50$ años) y el estado pluripatológico de los pacientes con HepC, en 
los que se hace necesario el uso de múltiples medicamentos para tratar las enfermedades coexistentes.

La polifarmacia puede aumentar la susceptibilidad a problemas relacionados con medicamentos (PRM) como RAM, caídas, reingresos hospitalarios, interacciones, entre otros (29), lo que hace necesaria la instauración de programas integrales de atención en salud en los que se incluya el seguimiento farmacoterapéutico con el fin de prevenir y resolver dichos PRM.

\section{CONCLUSIONES}

Se realizó la caracterización de los pacientes con HepC atendidos en el HPTU durante el período de estudio, en la que se encontró una distribución similar entre hombres y mujeres, con mayor prevalencia entre los 40-70 años, cuyo mecanismo de transmisión más frecuente es el transfusional. Los AAD presentaron mayor efectividad y seguridad en comparación con los esquemas con peg-IFN/RBV; $\sin$ embargo, el uso de RBV sigue siendo necesario en pacientes cirróticos con exposición previa a tratamiento, lo que aumenta el riesgo de aparición de RAM.

Se plantea la necesidad de implementar una atención integral centrada en el paciente que procure el acceso a los servicios de salud y medicamentos durante todo el curso del tratamiento, además del seguimiento farmacoterapéutico apropiado. Asimismo, son necesarios estudios prospectivos que evalúen los resultados de seguridad/efectividad de los $\mathrm{AAD}$ en los pacientes con HepC crónica.

\section{LIMITACIONES}

Este estudio presenta varias limitaciones dado su carácter retrospectivo, directamente dependiente de la calidad de la información registrada en la HCE. Durante la recolección de datos se detectaron registros incompletos, lo cual pudo atenuarse por el amplio período del estudio. Asimismo, se encontró una falta de uniformidad entre las notas médicas, lo que hace necesaria una estandarización de registros en la HCE que incluya el reporte de las fechas de inicio y terminación de tratamiento, las cargas virales, los tratamientos concomitantes y los posibles mecanismos de transmisión, con el fin de llevar un seguimiento adecuado del paciente y del reporte epidemiológico nacional.

\section{Agradecimientos}

Al grupo de investigación Promoción y Prevención Farmacéutica de la Universidad de Antioquia por el apoyo académico; y a la Unidad de Hepatología del HPTU por permitir el desarrollo de esta investigación.

\section{Conflicto de intereses}

Los autores declaran no tener ningún conflicto de intereses.

\section{Financiación}

El grupo Promoción y Prevención Farmacéutica recibió financiación de la convocatoria de sostenibilidad 20182019 del Comité para el Desarrollo de la Investigación de la Universidad de Antioquia.

\section{REFERENCIAS}

1. Hajarizadeh B, Grebely J, Dore GJ. Epidemiology and natural history of HCV infection. Nat Rev Gastroenterol Hepatol. 2013 Sep;10(9):553-62. doi: 10.1038/nrgastro.2013.107.

2. Kohli A, Shaffer A, Sherman A, Kottilil S. Treatment of hepatitis C: a systematic review. JAMA. 2014 Aug 13;312(6):631-40. doi: 10.1001/jama.2014.7085.

3. Center for Disease Analysis. Hepatitis C prevalence [Internet]. 2012 [acceso 19 de febrero de 2017]. Disponible en: http://www.centerforda.com/HepC/HepMap.html

4. American Association for the Study of Liver Diseases and the Infectious Diseases Society of America. HCV Guidance: Recommendations for Testing, Managing, and Treating Hepatitis C [Internet]. 2016 [acceso 23 de marzo de 2017]. Disponible en: http://www.hcvguidelines.org/full-report-view

5. Ministerio de Salud y Protección Social, Instituto de Evaluación Tecnológica en Salud. Guía de Práctica Clínica para la tamización, diagnóstico y tratamiento de personas con infección por el virus de la hepatitis C. Bogotá, Colombia: Ministerio de Salud y Protección Social; 2016.

6. Strader DB, Seeff LB. A brief history of the treatment of viral hepatitis C. Clin Liver Dis (Hoboken). 2012 Mar 6;1(1):611. doi: $10.1002 /$ cld.1.

7. Calleja JL, Crespo J, Rincón D, Ruiz-Antorán B, Fernandez I, Perelló C, et al. Effectiveness, safety and clinical outcomes of direct-acting antiviral therapy in HCV genotype 1 infection: Results from a Spanish real-world cohort. J Hepatol. 2017 Jun;66(6):1138-1148. doi: 10.1016/j.jhep.2017.01.028.

8. Yang S, Britt RB, Hashem MG, Brown JN. Outcomes of Pharmacy-Led Hepatitis C Direct-Acting Antiviral Utilization Management at a Veterans Affairs Medical Center. J Manag Care Spec Pharm. 2017 Mar;23(3):364369. doi: 10.18553/jmcp.2017.23.3.364.

9. Sistema de Trámites en Línea - Consultas Públicas [Internet]. [acceso 30 de julio de 2018]. Disponible en: http: / consultaregistro.invima.gov.co:8082/Consultas/ consultas/consreg_encabcum.jsp

10. Santos Ó, Gómez A, Vizcaíno V, Casas MC, Ramírez MDP, Olaya P. [Hepatitis $\mathrm{C}$ virus genotypes circulating in Colombia]. Biomedica. 2017 Jan 24;37(1):22-27. doi: 10.7705/biomedica.v37i1.3173. 
11. Yepes I de J, Carmona ZA, Múnera MN. Calidad de vida en pacientes con hepatitis $\mathrm{C}$ crónica en Colombia. Rev Colomb Gastroenterol. 2017;32(2):112. doi: 10.22516/25007440.139.

12. Yepes I de J, Lince B, Caez C, Vuono G de. Factores de riesgo para la infección por el virus de la hepatitis $\mathrm{C}$ en la Costa Caribe colombiana: un estudio de casos y controles. Biomédica. 2016;36(4):564-71. doi: 10.7705/biomédica. v36i4.3105.

13. Claudino Botero R, Tagle M. Los nuevos tratamiento de hepatitis C: Perspectivas latinoamericanas. Clin Liver Dis (Hoboken). 2015 Mar 4;5(1):11-13. doi: 10.1002/cld.466.

14. Beltrán $M$. Riesgo de infección transfusional de hepatitis $C$ en Colombia. Iatreia. 2004;17(3-S):305.

15. Saludes V, Ausina V, Martró E. Posibilidades actuales para predecir la respuesta a la terapia en pacientes con hepatitis C crónica por el genotipo 1 del virus de la hepatitis C. Enfermedades Infecc Microbiol Clínica. 2011;29:51-8. doi: 10.1016/S0213-005X(11)70044-1.

16. Buti M, Riveiro-Barciela M, Esteban R. Management of direct-acting antiviral agent failures. J Hepatol. 2015 Dec;63(6):1511-22. doi: 10.1016/j.jhep.2015.08.010.

17. Llerena S, Cabezas J, Iruzubieta P, Crespo J. Resistencias al virus de la hepatitis $C$. Implicaciones y posibilidades terapéuticas. Gastroenterol Hepatol. 2017;484-94. doi: 10.1016/j.gastrohep.2017.04.007.

18. Dietz J, Susser S, Berkowski C, Perner D, Zeuzem $S$, Sarrazin C. Consideration of Viral Resistance for Optimization of Direct Antiviral Therapy of Hepatitis C Virus Genotype 1-Infected Patients. PLoS One. 2015 Aug 28;10(8):e0134395. doi: 10.1371/journal.pone.0134395.

19. Sarrazin C, Dvory-Sobol H, Svarovskaia ES, Doehle B, Martin R, Zeuzem S, et al. The prevalence and the effect of HCV NS5A resistance associated variants in subjects with compensated cirrhosis treated with ledipasvir/sofosbuvir +/- RBV. J Hepatol. 2015;62:S620. doi: 10.1016/S01688278(15)30976-4.

20. Ministerio de Salud y Protección Social, Instituto de Evaluación Tecnológica en Salud. Vía clínica para el tratamiento de hepatitis C. Bogotá, Colombia: Ministerio de Salud y Protección Social; 2017. p. 41.
21. Santos OM, Orrego M. Tratamiento: Efectos adversos del tratamiento de hepatitis C. Rev Colomb Gastroenterol. 2012;27:37-40.

22. Mulet Pérez A, Pullés Labadié M, Gámez Escalona M, Mulet Gámez A, Díaz Santos O, Infante Velázquez M. Efectos adversos del tratamiento con interferón alfa-2b humano recombinante y rivabirina en pacientes con hepatitis crónica C. Rev Cuba Med Mil. 2011;40(1):76-84.

23. Sulkowski MS, Cooper C, Hunyady B, Jia J, Ogurtsov P, Peck-Radosavljevic M, Shiffman ML, Yurdaydin C, Dalgard O. Management of adverse effects of Peg-IFN and ribavirin therapy for hepatitis C. Nat Rev Gastroenterol Hepatol. 2011 Apr;8(4):212-23. doi: 10.1038/nrgastro.2011.21.

24. Huang YM, Wang $\mathrm{H}$, Wang $\mathrm{C}$, Chen $\mathrm{M}$, Zhao $\mathrm{MH}$. Promotion of hypercoagulability in antineutrophil cytoplasmic antibody-associated vasculitis by C5a-induced tissue factor-expressing microparticles and neutrophil extracellular traps. Arthritis Rheumatol. 2015 Oct;67(10):2780-90. doi: 10.1002/art.39239.

25. Hadziyannis SJ, Sette H Jr, Morgan TR, Balan V, Diago M, Marcellin P, Ramadori G, Bodenheimer H Jr, Bernstein D, Rizzetto M, Zeuzem S, Pockros PJ, Lin A, Ackrill AM; PEGASYS International Study Group. Peginterferonalpha2a and ribavirin combination therapy in chronic hepatitis C: a randomized study of treatment duration and ribavirin dose. Ann Intern Med. 2004 Mar 2;140(5):346-55. doi: 10.7326/0003-4819-140-5-200403020-00010.

26. Nachnani JS, Rao GA, Bulchandani D, Pandya PK, Alba LM. Predictors of hematological abnormalities in patients with chronic hepatitis $\mathrm{C}$ treated with interferon and ribavirin. Ann Hematol. 2010 Feb;89(2):121-5. doi: 10.1007/ s00277-009-0774-y.

27. UpToDate Inc. Ribavirin (systemic): Drug information [Internet]. [acceso 10 de agosto de 2018]. Disponible en: http://www.uptodate.com

28. Barrajón L, Soler E, Lorente L, Pérez J. Efectividad y seguridad de los antivirales de acción directa frente al virus de la hepatitis C. Rev OFIL. 2016;26(4):243-50.

29. Masnoon N, Shakib S, Kalisch-Ellett L, Caughey GE. What is polypharmacy? A systematic review of definitions. BMC Geriatr. 2017 Oct 10;17(1):230. doi: 10.1186/s12877-0170621-2. 